University of Nebraska - Lincoln

DigitalCommons@University of Nebraska - Lincoln

US Department of Energy Publications

U.S. Department of Energy

2010

\title{
Valence-shell photoionization of the chlorinelike $\mathrm{Ca} 3+$ ion
}

Ghassan A. Alna'washi

The Hashemite University, alnawashi@hu.edu.jo

M. Lu

University of Nevada - Reno, minggenl@unr.edu

M. Habibi

University of Nevada - Reno

R. A. Phaneuf

University of Nevada - Reno, phaneuf@unr.edu

A. L. D. Kilcoyne

Lawrence Berkeley National Laboratory, ALKilcoyne@lbl.gov

See next page for additional authors

Follow this and additional works at: https://digitalcommons.unl.edu/usdoepub

Part of the Bioresource and Agricultural Engineering Commons

Alna'washi, Ghassan A.; Lu, M.; Habibi, M.; Phaneuf, R. A.; Kilcoyne, A. L. D.; Schlachter, A. S.; Cisneros, C.; and McLaughlin, B. M., "Valence-shell photoionization of the chlorinelike Ca3+ ion" (2010). US Department of Energy Publications. 313.

https://digitalcommons.unl.edu/usdoepub/313

This Article is brought to you for free and open access by the U.S. Department of Energy at DigitalCommons@University of Nebraska - Lincoln. It has been accepted for inclusion in US Department of Energy Publications by an authorized administrator of DigitalCommons@University of Nebraska - Lincoln. 


\section{Authors}

Ghassan A. Alna'washi, M. Lu, M. Habibi, R. A. Phaneuf, A. L. D. Kilcoyne, A. S. Schlachter, C. Cisneros, and B. M. McLaughlin 


\title{
Valence-shell photoionization of the chlorinelike $\mathrm{Ca}^{3+}$ ion
}

\author{
Ghassan A. Alna'washi, ${ }^{*}$ M. Lu, M. Habibi, and R. A. Phaneuf \\ Department of Physics, MS-220, University of Nevada, Reno, Nevada 89557, USA
}

A. L. D. Kilcoyne and A. S. Schlachter

Advanced Light Source, Lawrence Berkeley National Laboratory, One Cyclotron Road, MS 7-100, University of California, Berkeley, California 94720, USA

C. Cisneros

Instituto de Ciencias Físicas, Universidad Nacional Autónoma de México, Apartado Postal 48-3, Cuernavaca 62210, México

\author{
B. M. McLaughlin \\ Centre for Theoretical Atomic, Molecular and Optical Physics (CTAMOP) School of Mathematics \& Physics, Queen's University Belfast, \\ The David Bates Building, 7 College Park, Belfast BT7 1NN, United Kingdom and \\ Institute for Theoretical Atomic and Molecular Physics (ITAMP) Harvard Smithsonian Center for Astrophysics, MS-14 Cambridge, \\ Massachusetts 02138, USA
}

(Received 24 December 2009; published 20 May 2010)

\begin{abstract}
Absolute photoionization measurements were performed for a mixture of ${ }^{2} P_{3 / 2}^{o}$ ground-state and ${ }^{2} P_{1 / 2}^{o}$ metastable-state $\mathrm{Ca}^{3+}$ ions over the photon energy range $65.7-104.6 \mathrm{eV}$ by merging an ion beam with a beam of monochromatized synchrotron radiation. The ionization threshold energy of the ${ }^{2} P_{3 / 2}^{o}$ ground state was measured to be $67.063 \pm 0.015 \mathrm{eV}$, which is $0.207 \mathrm{eV}$ lower than the value tabulated in the NIST database. Most of the observed resonances associated with multiple Rydberg series of autoionizing states have been assigned spectroscopically using the quantum defect form of the Rydberg formula, guided by relativistic Hartree-Fock calculations of resonance energies and oscillator strengths. Intermediate coupling $R$-matrix calculations performed using the semi-relativistic Breit-Pauli approximation are in suitable agreement with measured absolute photoionization cross section in the energy range studied for this complex Cl-like ion species.

DOI: 10.1103/PhysRevA.81.053416

PACS number(s): 32.80.Fb, 32.80.Aa, 32.70.Cs
\end{abstract}

\section{INTRODUCTION}

Photoionization of atomic ions and molecules using synchrotron radiation provides a probe of fundamental atomic interactions at an unprecedented level of precision [1]. Since most of the matter in the universe exists in ionic form and much of the information about the universe is carried by photons, the photoionization of ions is important in astrophysics [2]. The process plays a key role in high-temperature plasma environments such as those occurring in the stars and nebulae [3] as well as those in controlled thermonuclear fusion devices [4].

Systematic studies along isoelectronic sequences are useful in characterizing electron-electron interactions and in making predictions for other members of the sequence. Strong electron-electron interactions introduce complexity to the electronic structure of the chlorine isoelectronic sequence, and to date few experimental studies of photoionization of ions of the sequence have been reported. Kimura et al. [5] recorded He I photoelectron spectra for atomic chlorine. Ruscic and Berkowitz [6] reported a photoion-yield measurement for atomic chlorine from the ionization threshold to $750 \AA$ using synchrotron radiation. They observed three Rydberg series due to $3 p \rightarrow n s$ and $3 p \rightarrow n d$ excitations converging to the ${ }^{1} D_{2}$ and ${ }^{1} S_{0}$ states of $\mathrm{Cl}^{+}$. Their interpretation of these

*Current address: Department of Physics, The Hashemite University, Zarka, Jordan. measurements was revised by Hansen et al. [7]. Van der Meulin et al. [8] performed angle-resolved photoelectron spectroscopy of atomic chlorine using synchrotron radiation to study the $3 s 3 p^{5}\left({ }^{3} P_{2,1,0}^{o}\right) n p$ and $3 s 3 p^{5}\left({ }^{1} P_{1}^{o}\right) n p$ autoionizing Rydberg series. Krause et al. [9] reported $3 s$ and $3 p$ subshell photoionization spectra of chlorine atoms using highresolution photoelectron-spectrometry. A preliminary report was made by Covington et al. [10] of absolute photoionization cross-section measurements for Cl-like Argon $\left(\mathrm{Ar}^{+}\right)$ions based on the merged-beams technique (with synchrotron radiation). They assigned 17 Rydberg series due to $3 p \rightarrow n s$ and $3 p \rightarrow n d$ excitations converging to the ${ }^{1} D_{2}$ and ${ }^{1} S_{0}$ states of $\mathrm{Ar}^{2+}$.

Theoretically, photoionization of atomic chlorine has been studied extensively over the last few decades using a variety of approximations that include $R$-matrix or K-matrix calculations performed by several groups [11-14]. Photoionization crosssection calculations for atomic chlorine were also performed using the configuration-interaction (CI) method [15], manybody-perturbation theory (MBPT) [16], open-shell transition matrix [17], and an effective single particle potential [18]. We note that the $3 p$ photoionization cross section of Cl-like Potassium $\left(\mathrm{K}^{2+}\right)$ has been calculated using $R$-matrix theory [19] in $L S$-coupling where the presence of $3 s^{2} 3 p^{4}\left({ }^{1} D\right) n d$ and $3 s^{2} 3 p^{4}\left({ }^{1} S\right) n d$ Rydberg series were vividly illustrated. To our knowledge, no other theoretical photoionization cross section calculations have been reported on for any other members of this ionic sequence. 


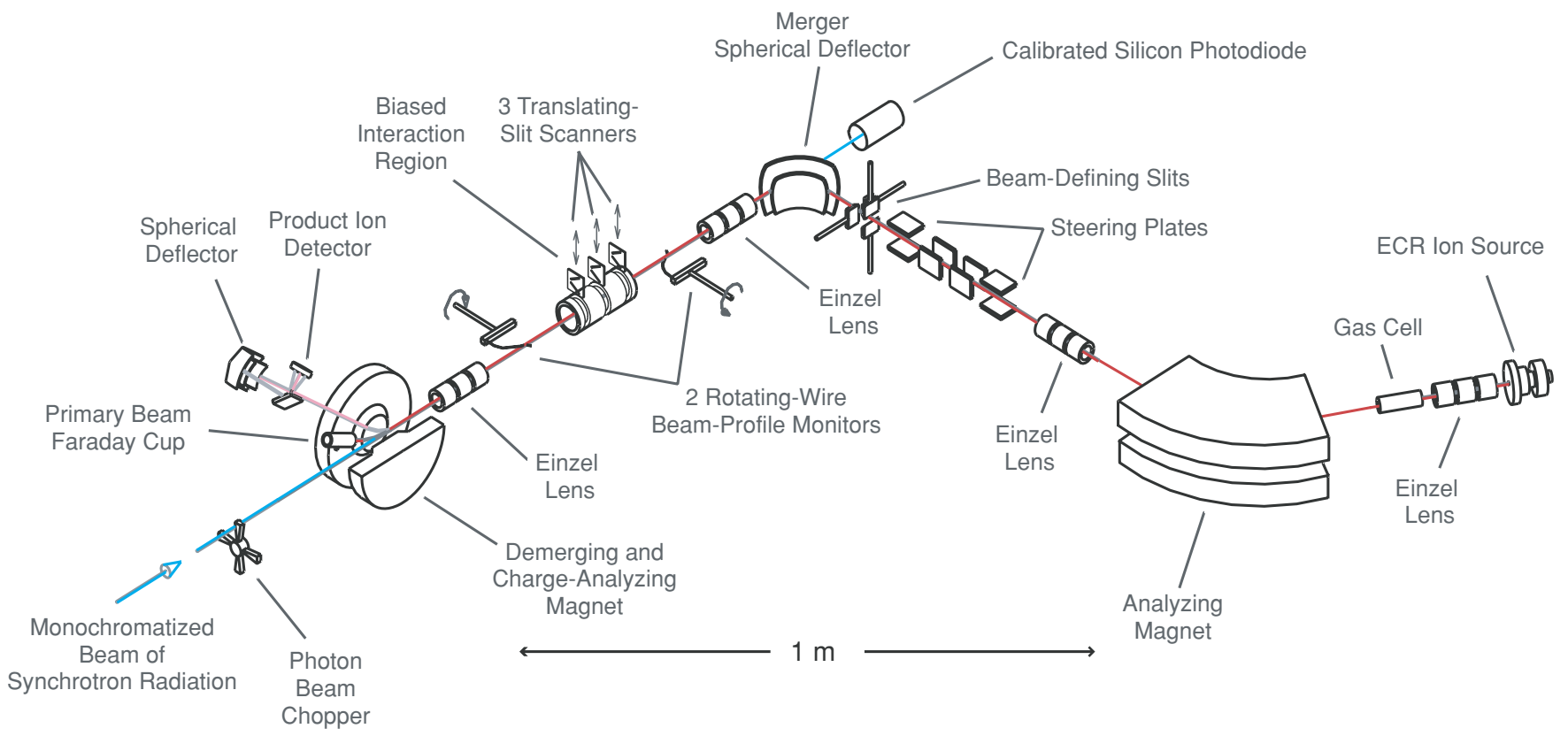

FIG. 1. (Color online) Schematic layout of the ion-photon merged-beams end station on ALS beamline 10.0.1.

In the present paper, absolute cross-section measurements for single photoionization of $\mathrm{Ca}^{3+}$ are presented in the energy range 65.7-106.6 eV. Most of the observed resonances are assigned spectroscopically using the quantum defect theory, guided by relativistic Hartree-Fock calculations of the energies and oscillator strengths of autoionizing transitions that are expected to contribute to photoionization. As part of this investigation the measurements are compared with theoretical calculations performed in intermediate coupling using the semi-relativistic Breit-Pauli $R$-matrix approximation for the photoionization cross section. Corresponding photoionization measurements and calculations for $\mathrm{Cl}$-like $\mathrm{K}^{2+}$ have been performed and are being prepared for publication.

\section{EXPERIMENT}

Absolute photoionization measurements were performed using the ion-photon merged-beams end station located at undulator beamline 10.0.1 of the Advanced Light Source. Figure 1 shows a schematic drawing of the experimental setup. A detailed description of this apparatus and measurement technique has been reported by Covington et al. [20]. Calcium evaporated from an oven was multiply ionized in a $10-\mathrm{GHz}$ permanent-magnet electron-cyclotron-resonance ion source. Ions were accelerated by a potential difference of $6 \mathrm{kV}$, focused by a series of cylindrical einzel lenses and magnetically analyzed according to their momentum per charge. A beam of ${ }^{40} \mathrm{Ca}^{3+}$ ions was selected, collimated and directed to a $90^{\circ}$ electrostatic deflector which merged it onto the axis of the photon beam. The latter was produced by an undulator and monochromatized by a grazing-incidence spherical-grating monochromator. A cylindrical einzel lens focused the primary beam in the center of the interaction region of length $29.4 \mathrm{~cm}$, to which an electrical potential of $+2 \mathrm{kV}$ was applied to energy-label $\mathrm{Ca}^{4+}$ product ions produced therein. Twodimensional spatial profiles of the merged ion and photon beams were recorded by three translating-slit scanners located near the beginning, middle and end points of the interaction region and used to quantify the spatial overlap of the beams.

Product $\mathrm{Ca}^{4+}$ ions were separated from the primary $\mathrm{Ca}^{3+}$ beam by a $45^{\circ}$ demerger magnet. The primary ion beam was collected in an extended Faraday cup, while a spherical $90^{\circ}$ electrostatic deflector whose dispersion plane is perpendicular to that of the demerger magnet directed the product ions onto to a single-particle detector. Photoion-yield spectra were obtained by counting $\mathrm{Ca}^{4+}$ product ions and recording the primary ion and photon beam currents as the photon beam energy was scanned in small steps. The photon flux was measured by a calibrated silicon $\mathrm{x}$-ray photodiode.

Absolute photoionization cross-section measurements were performed at a number of discrete photon energies where resonant features were absent in the photoion-yield spectra. These measurements were then used to place the normalized photoion-yield spectra onto an absolute cross-section scale. The total absolute uncertainty of the photoionization crosssection measurements is estimated to be $\pm 22 \%$.

The photon energy scale was calibrated in a separate experiment using a gas cell to measure krypton $\left(3 d_{5 / 2}^{-1} 5 p\right.$ and $3 d_{5 / 2}^{-1} 6 p$ ) resonances at $91.200 \pm 0.010 \mathrm{eV}$ and $92.560 \pm$ $0.010 \mathrm{eV}$ [21], and the helium $\left(E_{2}\right.$ and $\left.E_{3}\right)$ resonance energies of the $n=2$ double excitation Rydberg series at $60.147 \pm$ $0.004 \mathrm{eV}$ and $63.658 \pm 0.004 \mathrm{eV}$ [22]. The resulting uncertainty in the photon energy scale for the current measurements is estimated to be $\pm 0.015 \mathrm{eV}$.

\section{THEORY}

\section{A. $R$-matrix calculations}

To assist the experimental endeavor, $R$-matrix [23] calculations for the photoionization (PI) cross sections of the $\mathrm{Ca}^{3+}$ ion were performed in intermediate coupling using an efficient parallel version of the $R$-matrix codes [24,25] within the 
confines of a semirelativistic Breit-Pauli approximation [26]. For the PI calculations on this system, $30 L S \pi$ states (58 $J \pi$ states) were included in the close-coupling expansion arising from the following: $n=3$ and $n=4$ states of the $\mathrm{Ca}^{4+}$ ion core, namely, $1 s^{2} 2 s^{2} 2 p^{6} 3 s^{2} 3 p^{4}\left[{ }^{3} P,{ }^{1} D,{ }^{1} S\right]$, $1 s^{2} 2 s^{2} 2 p^{6} 3 s 3 p^{5}\left[{ }^{1,3} P^{o}\right], 1 s^{2} 2 s^{2} 2 p^{6} 3 s^{2} 3 p^{3}\left({ }^{4} S^{o},{ }^{2} D^{o},{ }^{2} P^{o}\right) 3 d$ $\left.{ }^{3,5} D^{o},{ }^{1,3} S^{o},{ }^{1,3} P^{o},{ }^{1,3} D^{o},{ }^{1,3} F^{o},{ }^{1,3} G^{o},{ }^{1,3} P^{o},{ }^{1,3} D^{o},{ }^{1,3} F^{o}\right]$, $1 s^{2} 2 s^{2} 2 p^{6} 3 s^{2} 3 p^{3}\left({ }^{4} S^{o},{ }^{2} D^{o},{ }^{2} P^{o}\right) 4 s\left[{ }^{1,3} P^{o},{ }^{1,3} D^{o}, 3,5 S^{o}\right]$, and $1 s^{2} 2 s^{2} 2 p^{6} 3 p^{6}\left[{ }^{1} S\right]$. The orbital basis set used for the $\mathrm{Ca}^{4+}$ product ion was limited to $n=4$ in constructing the multiconfiguration-interaction wave functions used in this work. The Breit-Pauli approximation was used to calculate the $58 \mathrm{~J} \pi$ energies of the $\mathrm{Ca}^{4+}$ ion arising from the above $30 L S \pi$ states and all the subsequent $\mathrm{Ca}^{3+}$ PI cross sections. A minor shift (less than a few \%) of the theoretical energies of the $\mathrm{Ca}^{4+}$ product ion was made in order to be in agreement with very accurate relativistic Hartree-Fock calculation [27] that are within $1 \%$ of the available experimental thresholds from the NIST tabulation (many of which are unobserved). PI cross sections were calculated for both the ${ }^{2} P_{3 / 2}^{o}$ ground state and the ${ }^{2} P_{1 / 2}^{o}$ metastable state of $\mathrm{Ca}^{3+}$ in intermediate coupling. The scattering wave functions were generated by allowing double-electron promotions out of the $n=3$ shell of the $1 s^{2} 2 s^{2} 2 p^{6} 3 s^{2} 3 p^{5}$ base configuration into the orbital set employed. All the scattering calculations were performed with 20 continuum basis functions and a boundary radius of $\sim 10$ Bohr radii. In the case of the ${ }^{2} P_{3 / 2}^{o}$ initial ground state, the dipole selection rule requires the dipole transition matrices, $3 / 2^{o} \rightarrow 1 / 2^{e}, 3 / 2^{e}, 5 / 2^{e}$, to be calculated, whereas for the metastable ${ }^{2} P_{1 / 2}^{o}$ state only the dipole matrices for the transitions $1 / 2^{\circ} \rightarrow 1 / 2^{e}, 3 / 2^{e}$ are included. The Hamiltonian matrices for the $1 / 2^{\circ}, 3 / 2^{\circ}, 5 / 2^{e}, 3 / 2^{e}$, and $1 / 2^{e}$ symmetries were then calculated, where the entire range of $L S$ matrices that contribute to these $J \pi$ symmetries. For both the initial ${ }^{2} P_{3 / 2}^{o}$ ground state and the ${ }^{2} P_{1 / 2}^{o}$ metastable states the outer region electron-ion collision problem was solved (in the resonance region below and between all the thresholds) using an energy mesh of $5 \times 10^{-8} \mathrm{Ry}(\sim 0.68 \mu \mathrm{eV})$ to fully resolve all the fine resonance structure in the appropriate PI cross sections. The theoretical PI cross sections were statistically averaged and convoluted with a Gaussian of the same full-width at half-maximum (FWHM) as that of experiment (55 meV) to enable a direct comparison to be made with the measurements.

\section{B. Hartree-Fock calculations}

The Hartree-Fock approximation [28] assumes that each electron in the atom moves independently in the nuclear Coulomb field and the average field of the other electrons and so the $N$-electron wave function is just the product of $N$ one-electron spatial wave functions. As a guide in the assignment of resonant features in the measurements, the Cowan atomic structure code [29], which is based on the relativistic Hartree-Fock (HFR) approximation, was used to calculate the energies and strengths of excitations contributing to the photoionization cross section for $\mathrm{Ca}^{3+}$. The Cowan code is based on $L S$ coupling and accounts for configuration interactions.

In the calculation of all transitions, $3 s^{2} 3 p^{5}$ was selected as the initial configuration. The final configurations selected were
$3 s^{2} 3 p^{4} n s(8 \leqslant n \leqslant 20)$ for $3 p \rightarrow n s$ transitions, $3 s^{2} 3 p^{4} n d$ $(7 \leqslant n \leqslant 20)$ for $3 p \rightarrow n d$ transitions and $3 s 3 p^{5} n p \quad(4 \leqslant$ $n \leqslant 10)$ for $3 s \rightarrow n p$ transitions. To determine the contributions of these transitions to the ionization measurements, the decay rates of the final states were calculated and compared to the inverse of the ion flight times in the experiment.

\section{Comparisons with experiment}

In order to compare the results of the $R$-matrix calculations with the experimental data, a statistically weighted sum of calculated cross sections from the ground state and the metastable state was convoluted with a Gaussian function that simulates the experimental photon energy distribution. To compare with the broad energy-scan measurements, a Gaussian function of $55 \mathrm{meV}$ FWHM was used. The calculated ground-state cross section was multiplied by $2 / 3$ and the metastable-state cross section by $1 / 3$ and the two weighted cross sections were added together.

\section{EXPERIMENTAL RESULTS AND ANALYSIS}

\section{A. Ionization threshold}

For the $1 s^{2} 2 s^{2} 2 p^{5} 3 s^{2} 3 p^{5}$ ground-state configuration of $\mathrm{Ca}^{3+}$, the Russell-Saunders notation gives the terms ${ }^{2} P_{3 / 2}^{o}$ for the ground state and ${ }^{2} P_{1 / 2}^{o}$ for the metastable state. Figure 2 shows the photoionization cross section for $\mathrm{Ca}^{3+}$ measured with a photon energy resolution of $15 \mathrm{meV}$ near the ground-state ionization threshold. The measurements indicate a step corresponding to a threshold at $67.063 \pm 0.015 \mathrm{eV}$, which is lower than the tabulated value of $67.27 \mathrm{eV}$ in the National Institute of Standards and Technology (NIST) atomic spectra database [30] by $0.207 \mathrm{eV}$. Using the tabulated fine-structure splitting of $0.387 \mathrm{eV}$ between the ground state and the ${ }^{2} P_{1 / 2}^{o}$ metastable state [28], the ionization threshold of the latter is predicted to be $66.676 \pm 0.015 \mathrm{eV}$. The measured photoionization below the ${ }^{2} P_{3 / 2}^{o}$ threshold is attributed to photoionization from the ${ }^{2} P_{1 / 2}^{o}$ metastable state.

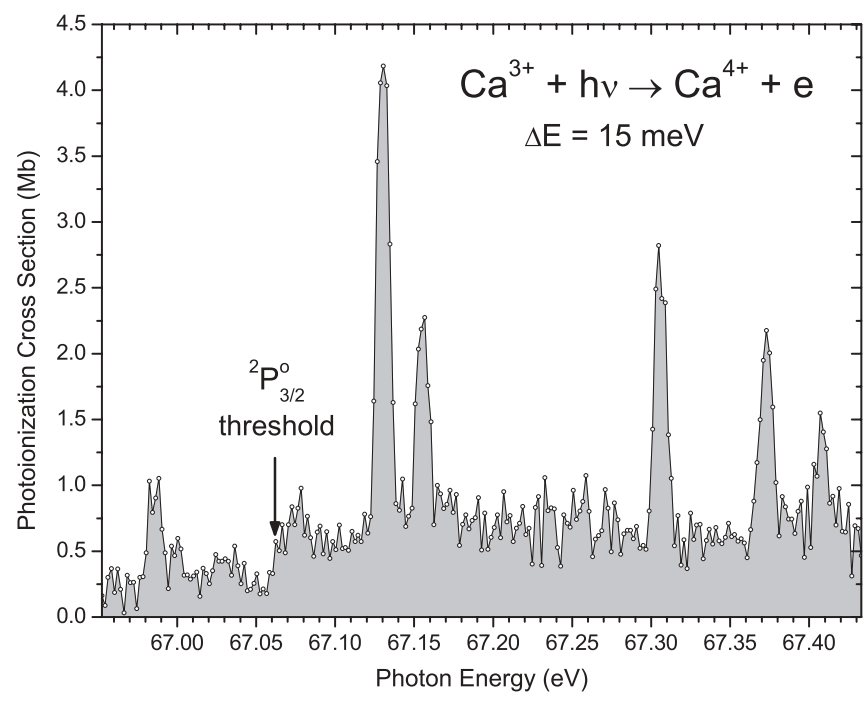

FIG. 2. Photoionization measurements at $15 \mathrm{meV}$ resolution near the ${ }^{2} P_{3 / 2}^{o}$ ground-state ionization threshold of $\mathrm{Ca}^{3+}$ measured at $67.063 \mathrm{eV}$. 


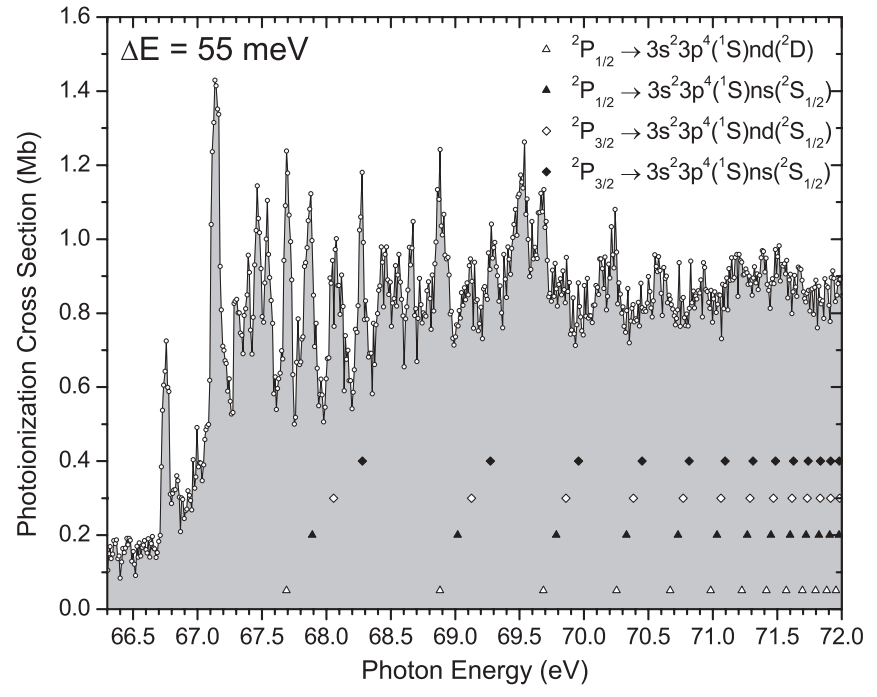

FIG. 3. Absolute photoionization cross-section measurements at a photon energy resolution of $55 \mathrm{meV}$ for $\mathrm{Ca}^{3+}$ from below the ${ }^{2} P_{1 / 2}^{o}$ metastable-state threshold to $72 \mathrm{eV}$. Four Rydberg series converging to ${ }^{1} S$ limit of $\mathrm{Ca}^{4+}$ are identified.

\section{B. Outer-shell $3 p \rightarrow n s$ and $3 p \rightarrow n d$ transitions}

Measurements in the energy region above the ${ }^{2} P_{1 / 2}^{o}$ and ${ }^{2} P_{3 / 2}^{o}$ thresholds are shown in Figs. 3 and 4. In this region 12 Rydberg series are characterized and assigned spectroscopi-

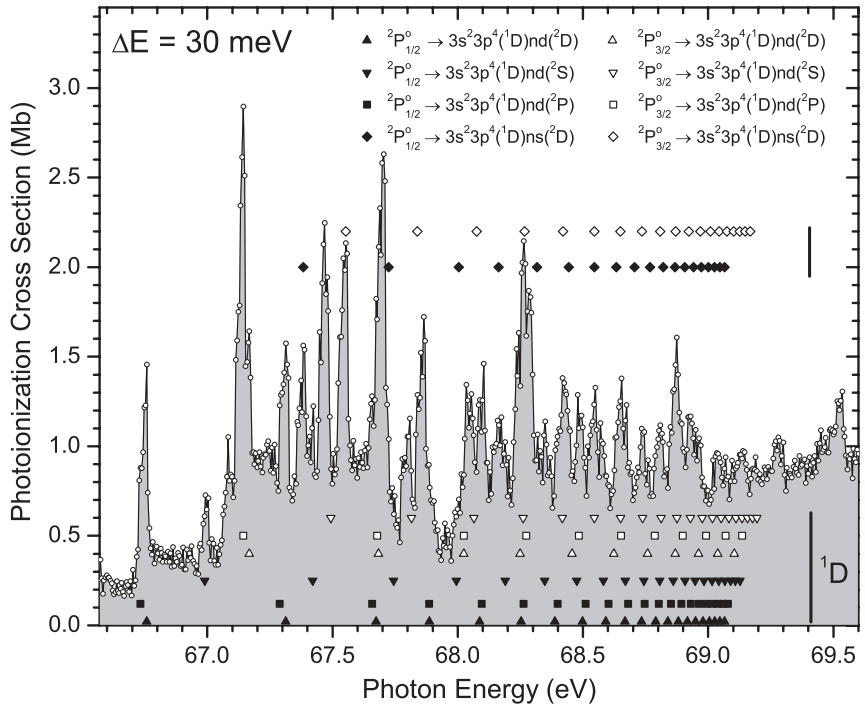

FIG. 4. Absolute photoionization cross-section measurements at a photon energy resolution of $30 \mathrm{meV}$ for $\mathrm{Ca}^{3+}$ from the ${ }^{2} P_{1 / 2}^{o}$ metastable state threshold to the ${ }^{1} D$ limit of $\mathrm{Ca}^{4+}$ (vertical line at $69.405 \mathrm{eV}$ ). Eight Rydberg series of resonances converging to the ${ }^{1} D$ limit are indicated.

cally using the quantum defect theory. Eight series converge to the ${ }^{1} D$ limit of $\mathrm{Ca}^{4+}$, four of which originate from the ${ }^{2} P_{1 / 2}^{o}$

TABLE I. Resonance energies, quantum defects $\delta$ determined from the measurements, series limits, and assignments for the $3 s^{2} 3 p^{4}\left({ }^{1} D\right) n s, m d$ Rydberg series. The estimated uncertainty in the resonance energies is $\pm 0.015 \mathrm{eV}$.

\begin{tabular}{|c|c|c|c|}
\hline Energy (eV) & Quantum defect $\delta$ & Series limit $(\mathrm{eV})$ & Assignment \\
\hline 66.758 & 0.92 & 69.398 & ${ }^{2} P_{1 / 2}^{o} \rightarrow 3 s^{2} 3 p^{4}\left({ }^{1} D\right) 10 d\left({ }^{2} D\right)$ \\
\hline 67.314 & 0.78 & 69.398 & ${ }^{2} P_{1 / 2}^{o} \rightarrow 3 s^{2} 3 p^{4}\left({ }^{1} D\right) 11 d\left({ }^{2} D\right)$ \\
\hline 67.674 & 0.76 & 69.398 & ${ }^{2} P_{1 / 2}^{o} \rightarrow 3 s^{2} 3 p^{4}\left({ }^{1} D\right) 12 d\left({ }^{2} D\right)$ \\
\hline 66.733 & 0.96 & 69.398 & ${ }^{2} P_{1 / 2}^{o} \rightarrow 3 s^{2} 3 p^{4}\left({ }^{1} D\right) 10 d\left({ }^{2} P\right)$ \\
\hline 67.289 & 0.84 & 69.398 & ${ }^{2} P_{1 / 2}^{o} \rightarrow 3 s^{2} 3 p^{4}\left({ }^{1} D\right) 11 d\left({ }^{2} P\right)$ \\
\hline 67.659 & 0.81 & 69.398 & ${ }^{2} P_{1 / 2}^{o} \rightarrow 3 s^{2} 3 p^{4}\left({ }^{1} D\right) 12 d\left({ }^{2} P\right)$ \\
\hline 66.993 & 0.49 & 69.398 & ${ }^{2} P_{1 / 2}^{o} \rightarrow 3 s^{2} 3 p^{4}\left({ }^{1} D\right) 10 d\left({ }^{2} S\right)$ \\
\hline 67.424 & 0.50 & 69.398 & ${ }^{2} P_{1 / 2}^{o} \rightarrow 3 s^{2} 3 p^{4}\left({ }^{1} D\right) 11 d\left({ }^{2} S\right)$ \\
\hline 67.739 & 0.55 & 69.398 & ${ }^{2} P_{1 / 2}^{o} \rightarrow 3 s^{2} 3 p^{4}\left({ }^{1} D\right) 12 d\left({ }^{2} S\right)$ \\
\hline 67.384 & 0.60 & 69.398 & ${ }^{2} P_{1 / 2}^{o} \rightarrow 3 s^{2} 3 p^{4}\left({ }^{1} D\right) 11 s\left({ }^{2} D_{3 / 2}\right)$ \\
\hline 67.724 & 0.60 & 69.398 & ${ }^{2} P_{1 / 2}^{o} \rightarrow 3 s^{2} 3 p^{4}\left({ }^{1} D\right) 12 s\left({ }^{2} D_{3 / 2}\right)$ \\
\hline 68.004 & 0.50 & 69.398 & ${ }^{2} P_{1 / 2}^{o} \rightarrow 3 s^{2} 3 p^{4}\left({ }^{1} D\right) 13 s\left({ }^{2} D_{3 / 2}\right)$ \\
\hline 67.168 & 1.12 & 69.398 & ${ }^{2} P_{3 / 2}^{o} \rightarrow 3 s^{2} 3 p^{4}\left({ }^{1} D\right) 11 d\left({ }^{2} D\right)$ \\
\hline 67.683 & 0.73 & 69.398 & ${ }^{2} P_{3 / 2}^{o} \rightarrow 3 s^{2} 3 p^{4}\left({ }^{1} D\right) 12 d\left({ }^{2} D\right)$ \\
\hline 68.024 & 0.41 & 69.398 & ${ }^{2} P_{3 / 2}^{o} \rightarrow 3 s^{2} 3 p^{4}\left({ }^{1} D\right) 13 d\left({ }^{2} D\right)$ \\
\hline 67.143 & 1.17 & 69.398 & ${ }^{2} P_{3 / 2}^{o} \rightarrow 3 s^{2} 3 p^{4}\left({ }^{1} D\right) 11 d\left({ }^{2} P\right)$ \\
\hline 67.679 & 0.75 & 69.398 & ${ }^{2} P_{3 / 2}^{o} \rightarrow 3 s^{2} 3 p^{4}\left({ }^{1} D\right) 12 d\left({ }^{2} P\right)$ \\
\hline 68.024 & 0.41 & 69.398 & ${ }^{2} P_{3 / 2}^{o} \rightarrow 3 s^{2} 3 p^{4}\left({ }^{1} D\right) 13 d\left({ }^{2} P\right)$ \\
\hline 67.469 & 0.38 & 69.398 & ${ }^{2} P_{3 / 2}^{o} \rightarrow 3 s^{2} 3 p^{4}\left({ }^{1} D\right) 11 d\left({ }^{2} S\right)$ \\
\hline 67.802 & 0.32 & 69.398 & ${ }^{2} P_{3 / 2}^{o} \rightarrow 3 s^{2} 3 p^{4}\left({ }^{1} D\right) 12 d\left({ }^{2} S\right)$ \\
\hline 68.104 & 0.03 & 69.398 & ${ }^{2} P_{3 / 2}^{o} \rightarrow 3 s^{2} 3 p^{4}\left({ }^{1} D\right) 13 d\left({ }^{2} S\right)$ \\
\hline 67.554 & 0.13 & 69.398 & ${ }^{2} P_{3 / 2}^{o} \rightarrow 3 s^{2} 3 p^{4}\left({ }^{1} D\right) 11 s\left({ }^{2} D_{3 / 2}\right)$ \\
\hline 67.839 & 0.18 & 69.398 & ${ }^{2} P_{3 / 2} \rightarrow 3 s^{2} 3 p^{4}\left({ }^{1} D\right) 12 s\left({ }^{2} D_{3 / 2}\right)$ \\
\hline 68.034 & 0.37 & 69.398 & ${ }^{2} P_{3 / 2}^{o} \rightarrow 3 s^{2} 3 p^{4}\left({ }^{1} D\right) 13 s\left({ }^{2} D_{3 / 2}\right)$ \\
\hline
\end{tabular}


TABLE II. Resonance energies, quantum defects $\delta$ determined from the measurements, series limits, and assignments for the $3 s^{2} 3 p^{4}\left({ }^{1} S\right) n d$ Rydberg series. The estimated uncertainty in the resonance energies is $\pm 0.015 \mathrm{eV}$.

\begin{tabular}{lccc}
\hline \hline Energy $(\mathrm{eV})$ & Quantum defect, $\delta$ & Series limit $(\mathrm{eV})$ & Assignment \\
\hline 67.691 & 0.27 & 72.498 & ${ }^{2} P_{1 / 2}^{o} \rightarrow 3 s^{2} 3 p^{4}\left({ }^{1} S\right) 7 d\left({ }^{2} D\right)$ \\
68.882 & 0.24 & 72.498 & ${ }^{2} P_{1 / 2}^{o} \rightarrow 3 s^{2} 3 p^{4}\left({ }^{1} S\right) 8 d\left({ }^{2} D\right)$ \\
69.686 & 0.20 & 72.498 & ${ }^{2} P_{1 / 2}^{o} \rightarrow 3 s^{2} 3 p^{4}\left({ }^{1} S\right) 9 d\left({ }^{2} D\right)$ \\
67.880 & 0.13 & 72.498 & ${ }^{2} P_{1 / 2}^{o} \rightarrow 3 s^{2} 3 p^{4}\left({ }^{1} S\right) 7 d\left({ }^{2} S_{1 / 2}\right)$ \\
69.051 & 0.05 & 72.498 & ${ }^{2} P_{1 / 2}^{o} \rightarrow 3 s^{2} 3 p^{4}\left({ }^{1} S\right) 8 d\left({ }^{2} S_{1 / 2}\right)$ \\
69.766 & 0.07 & 72.498 & ${ }^{2} P_{1 / 2}^{o} \rightarrow 3 s^{2} 3 p^{4}\left({ }^{1} S\right) 9 d\left({ }^{2} S_{1 / 2}\right)$ \\
68.058 & 1.00 & 72.498 & ${ }^{2} P_{3 / 2}^{o} \rightarrow 3 s^{2} 3 p^{4}\left({ }^{1} S\right) 8 d\left({ }^{2} D\right)$ \\
69.121 & 0.97 & 72.498 & ${ }^{2} P_{3 / 2}^{o} \rightarrow 3 s^{2} 3 p^{4}\left({ }^{1} S\right) 9 d\left({ }^{2} D\right)$ \\
69.865 & 0.91 & 72.498 & ${ }^{2} P_{3 / 2}^{o} \rightarrow 3 s^{2} 3 p^{4}\left({ }^{1} S\right) 10 d\left({ }^{2} D\right)$ \\
68.277 & 0.82 & 72.498 & ${ }^{2} P_{3 / 2}^{o} \rightarrow 3 s^{2} 3 p^{4}\left({ }^{1} S\right) 8 d\left({ }^{2} S_{1 / 2}\right)$ \\
69.279 & 0.78 & 72.498 & ${ }^{2} P_{3 / 2}^{o} \rightarrow 3 s^{2} 3 p^{4}\left({ }^{1} S\right) 9 d\left({ }^{2} S_{1 / 2}\right)$ \\
69.954 & 0.75 & 72.498 & ${ }^{2} P_{3 / 2}^{o} \rightarrow 3 s^{2} 3 p^{4}\left({ }^{1} S\right) 10 d\left({ }^{2} S_{1 / 2}\right)$ \\
\hline
\end{tabular}

metastable state and four from the ${ }^{2} P_{3 / 2}^{o}$ ground state. Four additional series converge to the ${ }^{1} S$ limit of $\mathrm{Ca}^{4+}$.

The four Rydberg series indicated in Fig. 3 converge to the ${ }^{1} S$ limit of $\mathrm{Ca}^{4+}$. Two of these series originate from the metastable state, one due to $3 p \rightarrow n d$ transitions (open triangles) and the other to $3 p \rightarrow n s$ transitions (filled triangles). The other two series originate from the ground state, one due to $3 p \rightarrow$ nd transitions (open diamonds) and the other to $3 p \rightarrow n s$ transitions (filled diamonds). None of the members of these series are separated enough to be resolved as distinct features at this energy resolution.

Of the four series indicated in Fig. 4 originating from the metastable state and converging to the ${ }^{1} D$ limit, three are due to $3 p \rightarrow n d$ transitions differing in their final coupling between the excited electron and the core. The $3 s^{2} 3 p^{4}\left({ }^{1} D\right) n d\left({ }^{2} D^{o}\right)$ (filled triangles) and $3 s^{2} 3 p^{4}\left({ }^{1} D\right) n d\left({ }^{2} P^{o}\right)$ (filled squares) are not separated enough to be resolved from each other. Their lower $n$ members $(n=10$ and $n=11)$ are distinct from the other Rydberg series but for higher $n$ they are not resolved as distinct features. The corresponding series originating from the ground state (open triangles and open squares) are also not separated enough to be resolved from each other and only their lower $n$ member $(n=11)$ is resolved as a distinct feature. The third series is attributed to $3 p \rightarrow n d$ transitions from the metastable state to $3 s^{2} 3 p^{4}\left({ }^{1} D\right) n d\left({ }^{2} S\right.$ ) (filled inverted triangles). The first two members of this series ( $n=10$ and $n=11$ ) are resolved from other series. For the corresponding series originating from the ground state (open inverted triangles), only the first member $(n=11)$ is resolved as a distinct feature. The final state for the fourth series originating from the metastable state is believed to be $3 s^{2} 3 p^{4}\left({ }^{1} D\right) n s\left({ }^{2} D\right)$ (filled diamonds). Only the first member of that series $(n=11)$ is distinct and resolved from other series. Similarly, for the corresponding series originating from the ground state, only the first member $(n=11)$ is a distinct feature.

These 12 Rydberg series originating from the ${ }^{2} P_{1 / 2}^{o}$ metastable state and the ${ }^{2} P_{3 / 2}^{o}$ ground state are grouped and listed in Tables I and II by the principal quantum number $n$ of the final state, energy position, and quantum defect parameter $\delta$. The quantum defects are based on $R_{Y}=13.6053 \mathrm{eV}$, the nuclear charge of $\mathrm{Ca}^{3+}(Z=20)$ and the series limit based on the value of the ionization threshold obtained from this experiment $\left(E_{\infty}=67.063 \mathrm{eV}\right)$.

\section{Inner-shell $3 s \rightarrow n p$ transitions}

Identified in Fig. 5 are series of resonances due to $3 s \rightarrow n p$ inner-shell excitation. Three Rydberg series, $3 s 3 p^{5}\left({ }^{3} P_{2}^{o}\right) n p$, $3 s 3 p^{5}\left({ }^{3} P_{1}^{o}\right) n p$, and $3 s 3 p^{5}\left({ }^{3} P_{0}^{o}\right) n p$, from the ${ }^{2} P_{1 / 2}^{o}$ metastable state and three corresponding series from the ${ }^{2} P_{3 / 2}^{o}$ ground state converging to the limits of $86.240 \mathrm{eV}, 86.499 \mathrm{eV}$, and $86.640 \mathrm{eV}$, respectively, are assigned. These six Rydberg series due to $3 s \rightarrow n p$ transitions are grouped and represented in Table III by their principal quantum number $n$, energy position, and quantum defect parameter $\delta$. The quantum defects are based on $R_{Y}=13.6053 \mathrm{eV}$, the nuclear charge of $\mathrm{Ca}^{3+}$ $(Z=20)$ and the series limit based on the value of the

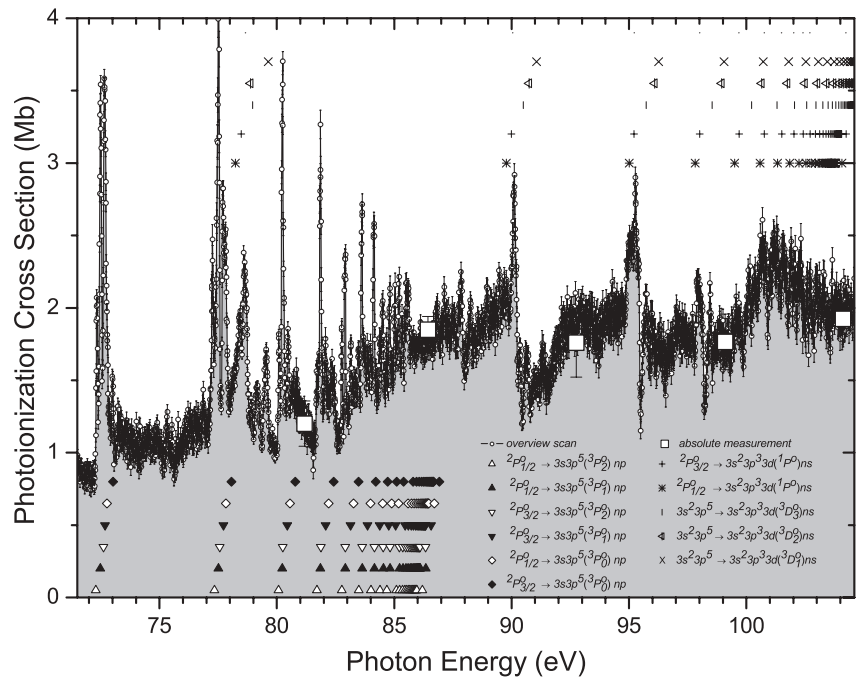

FIG. 5. Absolute photoionization cross-section measurements for $\mathrm{Ca}^{3+}$ at a photon energy resolution of $55 \mathrm{meV}$ in the energy range from 71 to $104 \mathrm{eV}$. Six Rydberg series of resonances due to $3 s \rightarrow n p$ excitations converging to the ${ }^{3} P_{2}^{o},{ }^{3} P_{1}^{o}$, and ${ }^{3} P_{0}^{o}$ excited states of $\mathrm{Ca}^{4+}$ are indicated. Five additional Rydberg series due to $3 s^{2} 3 p^{5} \rightarrow 3 s^{2} 3 p^{3} 3 d n s$ two-electron excitations converging to the $3 s^{2} 3 p^{3} 3 d\left({ }^{1} P_{1}^{o}\right)$ and $3 s^{2} 3 p^{3} 3 d\left({ }^{3} D_{3,2,1}^{o}\right)$ excited states of $\mathrm{Ca}^{4+}$ are also indicated. 
TABLE III. Resonance energies, quantum defects $\delta$ determined from the measurements, series limits, and assignments for the $3 s 3 p^{5}\left({ }^{3} P_{2,1,0}^{o}\right) n p$ Rydberg series. The estimated uncertainty in the resonance energies is $\pm 0.015 \mathrm{eV}$.

\begin{tabular}{|c|c|c|c|}
\hline Energy $(\mathrm{eV})$ & $\begin{array}{l}\text { Quantum } \\
\text { defect } \delta\end{array}$ & $\begin{array}{l}\text { Series limit } \\
\quad(\mathrm{eV})\end{array}$ & Assignment \\
\hline 72.327 & 0.04 & 86.240 & ${ }^{2} P_{1 / 2}^{o} \rightarrow 3 s 3 p^{5}\left({ }^{3} P_{2}\right) 4 p$ \\
\hline 77.255 & 0.07 & 86.240 & ${ }^{2} P_{1 / 2}^{o} \rightarrow 3 s 3 p^{5}\left({ }^{3} P_{2}\right) 5 p$ \\
\hline 80.089 & 0.05 & 86.240 & ${ }^{2} P_{1 / 2}^{o} \rightarrow 3 s 3 p^{5}\left({ }^{3} P_{2}\right) 6 p$ \\
\hline 81.741 & 0.04 & 86.240 & ${ }^{2} P_{1 / 2}^{o} \rightarrow 3 s 3 p^{5}\left({ }^{3} P_{2}\right) 7 p$ \\
\hline 82.772 & 0.08 & 86.240 & ${ }^{2} P_{1 / 2}^{o} \rightarrow 3 s 3 p^{5}\left({ }^{3} P_{2}\right) 8 p$ \\
\hline 83.493 & 0.10 & 86.240 & ${ }^{2} P_{1 / 2}^{o} \rightarrow 3 s 3 p^{5}\left({ }^{3} P_{2}\right) 9 p$ \\
\hline 72.495 & 0.06 & 86.499 & ${ }^{2} P_{1 / 2}^{o} \rightarrow 3 s 3 p^{5}\left({ }^{3} P_{1}\right) 4 p$ \\
\hline 77.476 & 0.08 & 86.499 & ${ }^{2} P_{1 / 2}^{o} \rightarrow 3 s 3 p^{5}\left({ }^{3} P_{1}\right) 5 p$ \\
\hline 80.249 & 0.10 & 86.499 & ${ }^{2} P_{1 / 2}^{o} \rightarrow 3 s 3 p^{5}\left({ }^{3} P_{1}\right) 6 p$ \\
\hline 81.861 & 0.15 & 86.499 & ${ }^{2} P_{1 / 2}^{o} \rightarrow 3 s 3 p^{5}\left({ }^{3} P_{1}\right) 7 p$ \\
\hline 82.923 & 0.20 & 86.499 & ${ }^{2} P_{1 / 2}^{o} \rightarrow 3 s 3 p^{5}\left({ }^{3} P_{1}\right) 8 p$ \\
\hline 83.634 & 0.28 & 86.499 & ${ }^{2} P_{1 / 2}^{o} \rightarrow 3 s 3 p^{5}\left({ }^{3} P_{1}\right) 9 p$ \\
\hline 72.773 & 0.04 & 86.640 & ${ }^{2} P_{1 / 2}^{o} \rightarrow 3 s 3 p^{5}\left({ }^{3} P_{0}\right) 4 p$ \\
\hline 77.816 & 0.03 & 86.640 & ${ }^{2} P_{1 / 2}^{o} \rightarrow 3 s 3 p^{5}\left({ }^{3} P_{0}\right) 5 p$ \\
\hline 80.500 & 0.05 & 86.640 & ${ }^{2} P_{1 / 2}^{o} \rightarrow 3 s 3 p^{5}\left({ }^{3} P_{0}\right) 6 p$ \\
\hline 82.302 & -0.08 & 86.640 & ${ }^{2} P_{1 / 2}^{o} \rightarrow 3 s 3 p^{5}\left({ }^{3} P_{0}\right) 7 p$ \\
\hline 83.233 & 0.01 & 86.640 & ${ }^{2} P_{1 / 2}^{o} \rightarrow 3 s 3 p^{5}\left({ }^{3} P_{0}\right) 8 p$ \\
\hline 72.644 & -0.001 & 86.240 & ${ }^{2} P_{3 / 2}^{o} \rightarrow 3 s 3 p^{5}\left({ }^{3} P_{2}\right) 4 p$ \\
\hline 77.526 & 0.002 & 86.240 & ${ }^{2} P_{3 / 2}^{o} \rightarrow 3 s 3 p^{5}\left({ }^{3} P_{2}\right) 5 p$ \\
\hline 80.249 & -0.03 & 86.240 & ${ }^{2} P_{3 / 2}^{o} \rightarrow 3 s 3 p^{5}\left({ }^{3} P_{2}\right) 6 p$ \\
\hline 81.861 & -0.05 & 86.240 & ${ }^{2} P_{3 / 2}^{o} \rightarrow 3 s 3 p^{5}\left({ }^{3} P_{2}\right) 7 p$ \\
\hline 82.973 & -0.16 & 86.240 & ${ }^{2} P_{3 / 2}^{o} \rightarrow 3 s 3 p^{5}\left({ }^{3} P_{2}\right) 8 p$ \\
\hline 83.634 & -0.14 & 86.240 & ${ }^{2} P_{3 / 2}^{o} \rightarrow 3 s 3 p^{5}\left({ }^{3} P_{2}\right) 9 p$ \\
\hline 72.694 & 0.03 & 86.499 & ${ }^{2} P_{3 / 2}^{o} \rightarrow 3 s 3 p^{5}\left({ }^{3} P_{1}\right) 4 p$ \\
\hline 77.706 & 0.02 & 86.499 & ${ }^{2} P_{3 / 2}^{o} \rightarrow 3 s 3 p^{5}\left({ }^{3} P_{1}\right) 5 p$ \\
\hline 80.460 & -0.004 & 86.499 & ${ }^{2} P_{3 / 2}^{o} \rightarrow 3 s 3 p^{5}\left({ }^{3} P_{1}\right) 6 p$ \\
\hline 82.072 & -0.01 & 86.499 & ${ }^{2} P_{3 / 2}^{o} \rightarrow 3 s 3 p^{5}\left({ }^{3} P_{1}\right) 7 p$ \\
\hline 83.153 & -0.07 & 86.499 & ${ }^{2} P_{3 / 2}^{o} \rightarrow 3 s 3 p^{5}\left({ }^{3} P_{1}\right) 8 p$ \\
\hline 73.002 & 0.005 & 86.640 & ${ }^{2} P_{3 / 2}^{o} \rightarrow 3 s 3 p^{5}\left({ }^{3} P_{0}\right) 4 p$ \\
\hline 78.097 & -0.05 & 86.640 & ${ }^{2} P_{3 / 2}^{o} \rightarrow 3 s 3 p^{5}\left({ }^{3} P_{0}\right) 5 p$ \\
\hline 80.850 & -0.13 & 86.640 & ${ }^{2} P_{3 / 2}^{o} \rightarrow 3 s 3 p^{5}\left({ }^{3} P_{0}\right) 6 p$ \\
\hline 82.352 & -0.13 & 86.640 & ${ }^{2} P_{3 / 2}^{o} \rightarrow 3 s 3 p^{5}\left({ }^{3} P_{0}\right) 7 p$ \\
\hline
\end{tabular}

ionization threshold obtained from this experiment $\left(E_{\infty}=\right.$ $67.063 \mathrm{eV})$.

For most of these series, the first eight members $(n=4$ to $n=11)$ are resolved. The measured resonances of these series
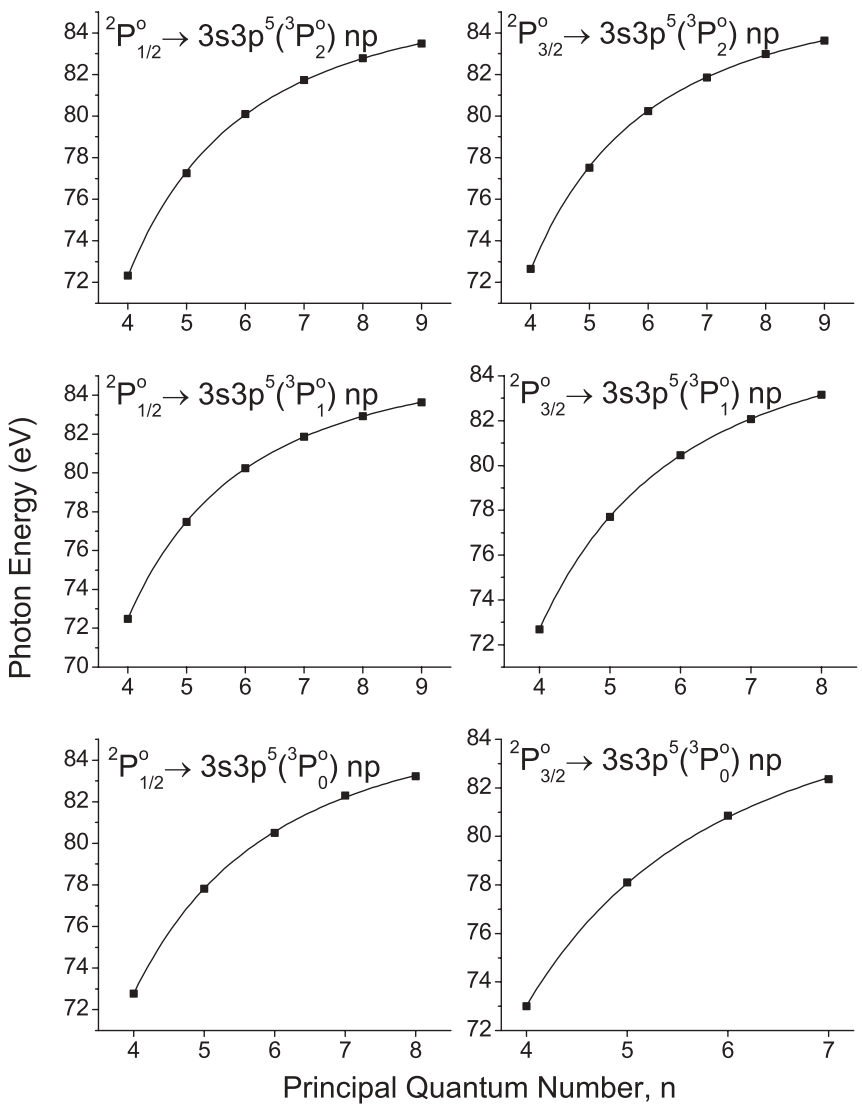

FIG. 6. Rydberg series fits for the $3 s 3 p^{5}\left({ }^{3} P_{2,1,0}^{o}\right) n p$ series originating from both the ground state and the metastable state.

are plotted versus the principal quantum number, $n$, as shown in Fig. 6 and fitted to the quantum defect form of the Rydberg formula [31] with the mean quantum defect parameter $(\delta)$ and the series limit $E_{\infty}$ as free parameters, while the Rydberg constant $\left(R_{Y}\right)$, nuclear charge $(Z)$, and the number of core electrons $\left(N_{c}\right)$ are fixed parameters. The fitted mean quantum defects $\delta_{\text {fit }}$ and series limits $E_{\infty}^{\text {fit }}$ for each series along with the series limits based on the ionization threshold obtained from the present experiment $E_{\infty}^{\text {expt }}$ and the series limit based on the tabulated ionization threshold $E_{\infty}^{\text {tab }}$ [30] are shown in Table IV. A comparison of the fitted series limits with the other two series limits provides additional evidence for the ground-state ionization threshold to be $67.063 \mathrm{eV}$.

An interesting question concerns the oscillator strengths of the assignment of $3 s 3 p^{5}\left({ }^{3} P_{2,1}^{o}\right) 4 p$ and $3 s 3 p^{5}\left({ }^{3} P_{2,1}^{o}\right) 5 p$

TABLE IV. Comparison between the generated series limits of the $3 s 3 p^{5}\left({ }^{3} P_{2,1,0}^{o}\right) n p$ and the series limits based on the ionization threshold obtained from this experiment and the tabulated ionization threshold [30].

\begin{tabular}{lccc}
\hline \hline Rydberg series & $\delta^{\mathrm{fit}}$ & $E_{\infty}^{\mathrm{fit}}(\mathrm{eV})$ & $E_{\infty}^{\exp }(\mathrm{eV})$ \\
\hline${ }^{2} P_{1 / 2}^{o} \rightarrow 3 s 3 p^{5}\left({ }^{3} P_{2}\right) n p$ & $0.045 \pm 0.009$ & $86.208 \pm 0.032$ & $86.240 \pm 0.015$ \\
${ }^{2} P_{1 / 2}^{o} \rightarrow 3 s 3 p^{5}\left({ }^{3} P_{1}\right) n p$ & $0.017 \pm 0.004$ & $86.376 \pm 0.014$ & $86.499 \pm 0.015$ \\
${ }^{2} P_{1 / 2}^{o} \rightarrow 3 s 3 p^{5}\left({ }^{3} P_{0}\right) n p$ & $0.049 \pm 0.014$ & $86.710 \pm 0.052$ & $86.640 \pm 0.015$ \\
${ }^{2} P_{3 / 2}^{o} \rightarrow 3 s 3 p^{5}\left({ }^{3} P_{2}\right) n p$ & $0.016 \pm 0.007$ & $86.337 \pm 0.024$ & $86.240 \pm 0.015$ \\
${ }^{2} P_{3 / 2}^{o} \rightarrow 3 s 3 p^{5}\left({ }^{3} P_{1}\right) n p$ & $0.041 \pm 0.003$ & $86.578 \pm 0.012$ & $86.499 \pm 0.015$ \\
${ }^{2} P_{3 / 2}^{o} \rightarrow 3 s 3 p^{5}\left({ }^{3} P_{0}\right) n p$ & $0.042 \pm 0.017$ & $86.921 \pm 0.068$ & $86.640 \pm 0.015$ \\
\hline \hline
\end{tabular}


TABLE V. Resonance energies, quantum defects determined from the measurements, series limits, and assignments for the $3 s^{2} 3 p^{3} 3 d\left({ }^{1} P\right) n s$ and $3 s^{2} 3 p^{3} 3 d\left({ }^{3} D\right) n s$ Rydberg series. The estimated uncertainty in the resonance energies is $\pm 0.015 \mathrm{eV}$.

\begin{tabular}{lccc}
\hline \hline & $\begin{array}{c}\text { Quantum } \\
\text { defect }\end{array}$ & $\begin{array}{c}\text { Series limit } \\
(\mathrm{eV})\end{array}$ & Assignment \\
\hline 78.399 & 1.114 & 104.529 & ${ }^{2} P_{1 / 2}^{o} \rightarrow 3 s^{2} 3 p^{3} 3 d\left({ }^{1} P_{1}^{o}\right) 4 s$ \\
89.928 & 1.139 & 104.529 & ${ }^{2} P_{1 / 2}^{o} \rightarrow 3 s^{2} 3 p^{3} 3 d\left({ }^{1} P_{1}^{o}\right) 5 s$ \\
95.142 & 1.184 & 104.529 & ${ }^{2} P_{1 / 2}^{o} \rightarrow 3 s^{2} 3 p^{3} 3 d\left({ }^{1} P_{1}^{o}\right) 6 s$ \\
97.927 & 1.258 & 104.529 & ${ }^{2} P_{1 / 2}^{o} \rightarrow 3 s^{2} 3 p^{3} 3 d\left({ }^{1} P_{1}^{o}\right) 7 s$ \\
99.404 & 1.483 & 104.529 & ${ }^{2} P_{1 / 2}^{o} \rightarrow 3 s^{2} 3 p^{3} 3 d\left({ }^{1} P_{1}^{o}\right) 8 s$ \\
78.650 & 1.100 & 104.529 & ${ }^{2} P_{3 / 2}^{o} \rightarrow 3 s^{2} 3 p^{3} 3 d\left({ }^{1} P_{1}^{o}\right) 4 s$ \\
90.120 & 1.113 & 104.529 & ${ }^{2} P_{3 / 2}^{o} \rightarrow 3 s^{2} 3 p^{3} 3 d\left({ }^{1} P_{1}^{o}\right) 5 s$ \\
95.360 & 1.127 & 104.529 & ${ }^{2} P_{3 / 2}^{o} \rightarrow 3 s^{2} 3 p^{3} 3 d\left({ }^{1} P_{1}^{o}\right) 6 s$ \\
98.128 & 1.168 & 104.529 & ${ }^{2} P_{3 / 2}^{o} \rightarrow 3 s^{2} 3 p^{3} 3 d\left({ }^{1} P_{1}^{o}\right) 7 s$ \\
99.564 & 1.379 & 104.529 & ${ }^{2} P_{3 / 2}^{o} \rightarrow 3 s^{2} 3 p^{3} 3 d\left({ }^{1} P_{1}^{o}\right) 8 s$ \\
78.908 & 1.127 & 105.273 & $3 s^{2} 3 p^{5} \rightarrow 3 s^{2} 3 p^{3} 3 d\left({ }^{3} D_{3}^{o}\right) 4 s$ \\
90.683 & 1.137 & 105.273 & $3 s^{2} 3 p^{5} \rightarrow 3 s^{2} 3 p^{3} 3 d\left({ }^{3} D_{3}^{o}\right) 5 s$ \\
95.709 & 1.229 & 105.273 & $3 s^{2} 3 p^{5} \rightarrow 3 s^{2} 3 p^{3} 3 d\left({ }^{3} D_{3}^{o}\right) 6 s$ \\
98.452 & 1.351 & 105.273 & $3 s^{2} 3 p^{5} \rightarrow 3 s^{2} 3 p^{3} 3 d\left({ }^{3} D_{3}^{o}\right) 7 s$ \\
79.168 & 1.124 & 105.477 & $3 s^{2} 3 p^{5} \rightarrow 3 s^{2} 3 p^{3} 3 d\left({ }^{3} D_{2}^{o}\right) 4 s$ \\
90.612 & 1.173 & 105.477 & $3 s^{2} 3 p^{5} \rightarrow 3 s^{2} 3 p^{3} 3 d\left({ }^{3} D_{2}^{o}\right) 5 s$ \\
95.979 & 1.213 & 105.477 & $3 s^{2} 3 p^{5} \rightarrow 3 s^{2} 3 p^{3} 3 d\left({ }^{3} D_{2}^{o}\right) 6 s$ \\
99.312 & 1.058 & 105.477 & $3 s^{2} 3 p^{5} \rightarrow 3 s^{2} 3 p^{3} 3 d\left({ }^{3} D_{2}^{o}\right) 7 s$ \\
79.548 & 1.110 & 105.615 & $3 s^{2} 3 p^{5} \rightarrow 3 s^{2} 3 p^{3} 3 d\left({ }^{3} D_{1}^{o}\right) 4 s$ \\
91.303 & 1.100 & 105.615 & $3 s^{2} 3 p^{5} \rightarrow 3 s^{2} 3 p^{3} 3 d\left({ }^{3} D_{1}^{o}\right) 5 s$ \\
96.290 & 1.168 & 105.615 & $3 s^{2} 3 p^{5} \rightarrow 3 s^{2} 3 p^{3} 3 d\left({ }^{3} D_{1}^{o}\right) 6 s$ \\
98.863 & 1.322 & 105.615 & $3 s^{2} 3 p^{5} \rightarrow 3 s^{2} 3 p^{3} 3 d\left({ }^{3} D_{1}^{o}\right) 7 s$ \\
\hline \hline & & & \\
\hline
\end{tabular}

resonances. It is believed that for $n$ values higher than 4 the ${ }^{2} P_{3 / 2}^{o} \rightarrow 3 s 3 p^{5}\left({ }^{3} P_{2}^{o}\right) n p$ resonances and ${ }^{2} P_{1 / 2}^{o} \rightarrow$ $3 s 3 p^{5}\left({ }^{3} P_{1}^{o}\right) n p$ resonances occur at photon energies close enough to be unresolved from one another.

Asymmetric Fano-Beutler line shapes [32] of the $3 s \rightarrow n p$ resonances are evident in Fig. 5, indicating strong interference between the direct and the indirect photoionization channels for excitation of the $3 s$ subshell.

\section{Two electron excitations: $3 p^{5} \rightarrow 3 p^{3} 3 d n s$}

Resonances due to two-electron excitations, $3 p^{5} \rightarrow$ $3 p^{3} 3 d n s$, are evident in Fig. 5. A Rydberg series, $3 s^{2} 3 p^{3} 3 d\left({ }^{1} P_{1}^{o}\right) n s$ originating from the ${ }^{2} P_{1 / 2}^{o}$ metastable state and a corresponding series originating from the ground state converging to the series limit of $104.529 \mathrm{eV}$ are assigned. Three
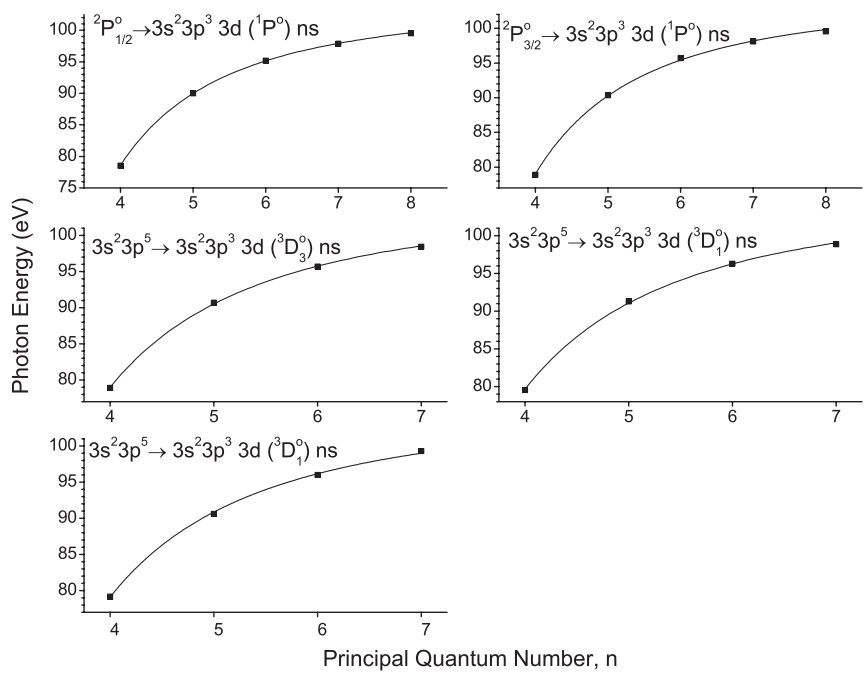

FIG. 7. Rydberg fits for $3 s^{2} 3 p^{3} 3 d\left({ }^{1} P\right) n s$ and $3 s^{2} 3 p^{3} 3 d\left({ }^{3} D\right) n s$ series originating from both the ground state and the metastable state.

more Rydberg series $3 s^{2} 3 p^{3} 3 d\left({ }^{3} D_{3}^{o}\right) n s, 3 s^{2} 3 p^{3} 3 d\left({ }^{3} D_{2}^{o}\right) n s$, and $3 s^{2} 3 p^{3} 3 d\left({ }^{3} D_{1}^{o}\right) n s$ converging to the series limits of $105.273 \mathrm{eV}, 105.477 \mathrm{eV}$, and $105.615 \mathrm{eV}$, respectively, are assigned. These five Rydberg series due to $3 p^{5} \rightarrow 3 p^{3} 3 d n s$ transitions are grouped and tabulated in Table $\mathrm{V}$ by their principal quantum numbers $n$, resonance energies, and quantum defect parameters $\delta$.

The measured resonances of these series are plotted versus the principal quantum number in Fig. 7 and fitted to the quantum defect form of the Rydberg formula with the mean quantum defect parameter $\delta$ and the series limit $E_{\infty}$ as free parameters. The Rydberg constant $R_{Y}$, nuclear charge $Z$, and the number of core electrons $N_{c}$ are fixed parameters. The fitted mean quantum defect $\delta^{\text {fit }}$ and the generated series limit $E_{\infty}^{\text {fit }}$ for each series along with the series limits based on ionization threshold obtained from the experiment $E_{\infty}^{\text {expt }}$ and the tabulated ionization threshold $E_{\infty}^{\mathrm{tab}}$ [30] are shown in Table VI. The comparison of the fitted series limits with the other two series limits based on the tabulated ionization threshold and the ionization threshold obtained from this experiment provides additional evidence that the ground-state ionization threshold is $67.063 \mathrm{eV}$.

\section{COMPARISON BETWEEN ABSOLUTE CROSS-SECTION MEASUREMENTS AND THEORY}

The absolute cross-section measurements taken at a photon energy resolution of $55 \mathrm{meV}$ are compared in Fig. 8 with the

TABLE VI. Comparison between the fitted series limits of the $3 s^{2} 3 p^{3} 3 d\left({ }^{1} P\right) n s$ and $3 s^{2} 3 p^{3} 3 d\left({ }^{3} D\right) n s$ and the series limits based on the ground-state ionization thresholds obtained from the present experiment and from the NIST-tabulated value [30].

\begin{tabular}{lccr}
\hline \hline Rydberg series & $\delta^{\mathrm{fit}}$ & $E_{\infty}^{\mathrm{fit}}(\mathrm{eV})$ & $E_{\infty}^{\mathrm{expt}}(\mathrm{eV})$ \\
\hline${ }^{2} P_{1 / 2}^{o} \rightarrow 3 s^{2} 3 p^{3} 3 d\left({ }^{1} P\right) n s$ & $1.081 \pm 0.004$ & $104.125 \pm 0.039$ & $104.529 \pm 0.015$ \\
${ }^{2} P_{3 / 2}^{o} \rightarrow 3 s^{2} 3 p^{3} 3 d\left({ }^{1} P\right) n s$ & $1.081 \pm 0.018$ & $104.340 \pm 0.158$ & $104.529 \pm 0.015$ \\
$3 \mathrm{~s}^{2} 3 p^{5} \rightarrow 3 s^{2} 3 p^{3} 3 d\left({ }^{3} D_{3}^{o}\right) n s$ & $1.097 \pm 0.012$ & $104.793 \pm 0.121$ & $105.273 \pm 0.015$ \\
$3 \mathrm{~s}^{2} 3 p^{5} \rightarrow 3 s^{2} 3 p^{3} 3 d\left({ }^{3} D_{2}^{o}\right) n s$ & $1.128 \pm 0.025$ & $105.257 \pm 0.254$ & $105.477 \pm 0.015$ \\
$3 \mathrm{~s}^{2} 3 p^{5} \rightarrow 3 s^{2} 3 p^{3} 3 \mathrm{~d}\left({ }^{3} D_{1}^{o}\right) n s$ & $1.086 \pm 0.019$ & $105.276 \pm 0.182$ & $105.615 \pm 0.015$ \\
\hline \hline
\end{tabular}




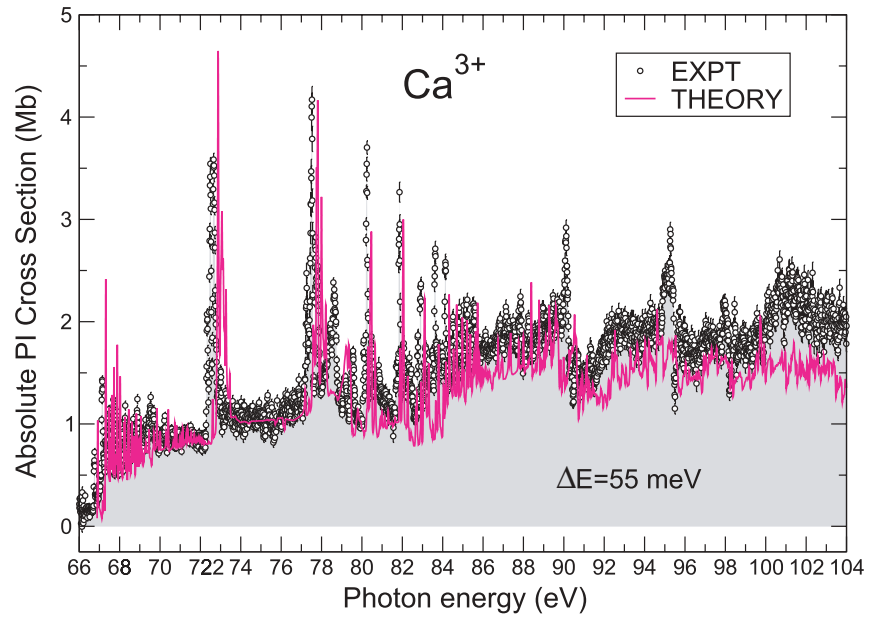

FIG. 8. (Color online) Comparison of theoretical Breit-Pauli $R$-matrix cross-section calculations (solid line) with absolute crosssection measurements (open circles) for photoionization of $\mathrm{Ca}^{3+}$ ions. The theoretical results have been convoluted with a Gaussian of $55 \mathrm{meV}$ FWHM to simulate the photon energy resolution of the measurement and weighted for a statistical initial-state admixture of the ${ }^{2} P_{3 / 2}^{o}$ ground state $(2 / 3)$ and ${ }^{2} P_{1 / 2}^{o}$ metastable state $(1 / 3)$.

results of the Breit-Pauli $R$-matrix theoretical calculation. As noted, the calculation represents a sum of the photoionization cross sections from the ground state and the metastable state multiplied by their statistical weights $(2 / 3$ and $1 / 3$, respectively). The comparison shows good agreement in the magnitudes of both the resonant and the nonresonant contributions to the photoionization cross section. The energy positions of the calculated resonances are slightly shifted from the experimental values. The theory also reproduces the observed Fano-Beutler asymmetric resonance lineshapes. The two-electron excitation processes, $3 p^{5} \rightarrow 3 p^{3} 3 d n s$, that result in broad resonance features in the measured cross section in the energy range 77.96-79.80 eV, are accounted for in the $R$-matrix calculation.

\section{SUMMARY}

The absolute photoionization cross section for a mixture of ${ }^{2} P_{3 / 2}^{o}$ ground-state and ${ }^{2} P_{1 / 2}^{o}$ metastable-state $\mathrm{Ca}^{3+}$ was measured in the photon energy range $65.7-104.6 \mathrm{eV}$ at a resolution of $55 \mathrm{meV}$ using merged beams and synchrotron radiation. The cross section is rich in resonance structure due to indirect photoionization. Guided by Hartree-Fock atomic-structure calculations, the main features have been spectroscopically assigned to Rydberg series using a quantum-defect analysis. From additional measurements performed at higher resolution, the ground-state ionization threshold was determined to be $67.063 \pm 0.015 \mathrm{eV}$, which is $0.207 \mathrm{eV}$ lower than the value tabulated in NIST database. Overall, the measurements are in suitable agreement with theoretical results obtained from a Breit-Pauli $R$-matrix calculation performed in intermediate coupling. Minor discrepancies between theory and experiment in the spectra of this complex $\mathrm{Cl}$-like ion are attributed to limitations of the $n=4$ basis set used in the calculations.

\section{ACKNOWLEDGMENTS}

The Division of Chemical Sciences, Geosciences, and Biosciences of the US Department of Energy supported the experimental research under Grant No. DE-FG02-03ER15424. B.M.M. acknowledges support by the US National Science Foundation through a grant to ITAMP at the HarvardSmithsonian Center for Astrophysics. The computational work was carried out at the National Energy Research Scientific Computing Center in Oakland, California, which is supported by the US Department of Energy, and on the Tera-grid at the National Institute for Computational Science (NICS) in Tennessee, which is supported in part by the US National Science Foundation. C.C. acknowledges support from PAPITUNAM IN109407-IN108009, Mexico.
[1] J. B. West, J. Phys. B 34, R45 (2001).

[2] D. R. Flower, in Atoms in Astrophysics, edited by P. G. Burke, W. B. Eissner, D. G. Hummer, and I. C. Percival (Plenum Press, New York, 1983), pp. 289-323.

[3] I. Hofmann, Laser Part. Beams 8, 527 (1990).

[4] R. W. P. McWhirter and H. P. Summers, in Applied Atomic Collision Physics, Vol. 2, "Plasma," edited by C. F. Barnett and M. F. Harrison (Academic Press, New York, 1983), pp. 51-111.

[5] K. Kimura, T. Yamazaki, and Y. Achiba, Chem. Phys. Lett. 58, 104 (1978).

[6] B. Ruscic and J. Berkowitz, Phys. Rev. Lett. 50, 675 (1983).

[7] J. E. Hansen, R. D. Cowan, S. L. Carter, and H. P. Kelly, Phys. Rev. A 30, 1540 (1984).

[8] P. van der Meulen, M. O. Krause, C. D. Caldwell, S. B. Whitfield, and C. A. de Lange, Phys. Rev. A 46, 2468 (1992).

[9] M. O. Krause, C. D. Caldwell, S. B. Whitfield, C. A. de Lange, and P. van der Meulen, Phys. Rev. A 47, 3015 (1993).

[10] A. M. Covington, A. Aguilar, I. Alvarez, C. Cisneros, I. R. Covington, I. Dominguez, M. F. Gharaibeh, G. Hinojosa, M. M. Sant'Anna, R. A. Phaneuf, and A. S. Schlachter, in Proceedings of the XXII International Conference on Photonic, Electronic and Atomic Collisions, edited by S. Datz, M. E. Bannister, H. F. Krause, L. H. Saddiq, D. R. Schultz, and C. R. Vane (Rinton Press, Princeton, NJ, 2001), p. 48.

[11] S. S. Tayal, Phys. Rev. A 47, 182 (1993).

[12] Z. Felfli, N. C. Deb, D. S. F. Crothers, and A. Z. Msezane, J. Phys. B 35, L419 (2002).

[13] F. Robicheaux and C. H. Greene, Phys. Rev. A 46, 3821 (1992).

[14] W. R. Fielder and L. Armstrong Jr., Phys. Rev. A 28, 218 (1983).

[15] M. Martins, J. Phys. B 34, 1321 (2001).

[16] J. A. R. Samson, Y. Shefer, and G. C. Angel, Phys. Rev. Lett. 56, 2020 (1986).

[17] S. Shahabi, A. F. Starace, and T. N. Chang, Phys. Rev. A 30, 1819 (1984).

[18] Z. D. Qian, S. L. Carter, and H. P. Kelly, Phys. Rev. A 33, 1751 (1986).

[19] F. Combet Farnoux, M. Lamoureux, and K. T. Taylor, J. Phys. B 11, 2855 (1978). 
[20] A. M. Covington, A. Aguilar, I. R. Covington, M. F. Gharaibeh, G. Hinojosa, C. A. Shirley, R. A. Phaneuf, I. Álvarez, C. Cisneros, I. Dominguez-Lopez, M. M. Sant'Anna, A. S. Schlachter, B. M. McLaughlin, and A. Dalgarno, Phys. Rev. A 66, 062710 (2002).

[21] G. C. King, M. Tronc, F. H. Read, and R. C. Bradford, J. Phys. B 10, 2479 (1977).

[22] M. Domke, K. Schulz, G. Remmers, G. Kaindl, and D. Wintgen, Phys. Rev. A 53, 1424 (1996).

[23] P. G. Burke and W. D. Robb, Advances in Atomic and Molecular Physics (Academic Press, New York, 1975), Vol. 11, pp. 143-214.

[24] C. P. Ballance and D. C. Griffin, J. Phys. B 39, 3617 (2006).

[25] C. P. Ballance, P. H. Norrington, and B. M. McLaughlin, J. Phys. $\mathrm{B}$ (in preparation).
[26] P. G. Burke and K. A. Berrington, Atomic and Molecular Processes: An R-matrix Approach (IOP Publishing, Bristol, UK, 1993).

[27] E. Biémont, Phys. Scr. 34, 318 (1986).

[28] H. Friedrich, Theoretical Atomic Physics, 2nd ed. (Springer, Berlin, 1998), pp. 85-92.

[29] R. D. Cowan, The Theory of Atomic Structure and Spectra (University of California Press, Berkeley, 1981).

[30] Y. Ralchenko, A. E. Kramida, J. Reader, and NIST ASD Team, NIST Atomic Spectra Database (version 3.1.4), National Institute of Standards and Technology, Gaithersburg, MD [http://physics.nist.gov/asd3].

[31] M. J. Seaton, Rep. Prog. Phys. 46, 167 (1983).

[32] U. Fano, Phys. Rev. 124, 1866 (1961). 